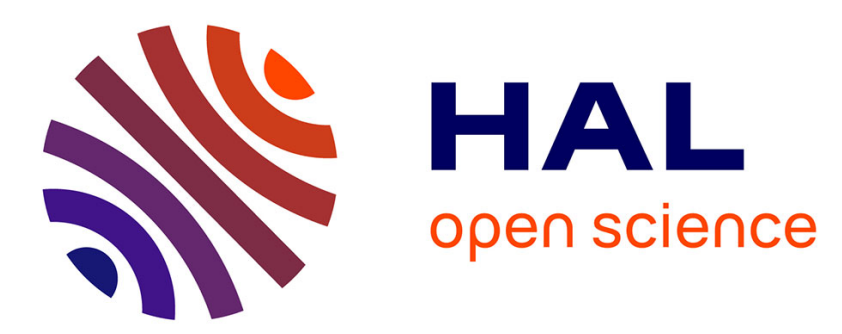

\title{
Force estimation in a piezoelectric cantilever using the Inverse-Dynamics-Based UIO technique.
}

\author{
Micky Rakotondrabe, Philippe Lutz
}

\section{To cite this version:}

Micky Rakotondrabe, Philippe Lutz. Force estimation in a piezoelectric cantilever using the InverseDynamics-Based UIO technique.. IEEE International Conference on Robotics and Automation, ICRA'09., May 2009, Kobe, Japan. 6 p. hal-00400748

\section{HAL Id: hal-00400748 https://hal.science/hal-00400748}

Submitted on 1 Jul 2009

HAL is a multi-disciplinary open access archive for the deposit and dissemination of scientific research documents, whether they are published or not. The documents may come from teaching and research institutions in France or abroad, or from public or private research centers.
L'archive ouverte pluridisciplinaire HAL, est destinée au dépôt et à la diffusion de documents scientifiques de niveau recherche, publiés ou non, émanant des établissements d'enseignement et de recherche français ou étrangers, des laboratoires publics ou privés. 


\title{
Force Estimation in a Piezoelectric Cantilever using the Inverse-Dynamics-Based UIO Technique
}

\author{
Micky Rakotondrabe, Member, IEEE and Philippe Lutz, Member, IEEE
}

\begin{abstract}
This paper presents the estimation of the force applied by a piezocantilever dedicated to micromanipulation/microassembly. Relative to previous works, the presented method avoids the reliance on the force dynamics on the characteristics of the microobjects. Furthermore, the estimation is a closed-loop kind technique so that convergency can be ensured efficiently. To perform these, we consider the force at the tip of a piezocantilever as an unknown input and we use an Unknown Input Observation technique. We especially use the Inverse-Dynamics-Based UIO technique because it is well suited for a piezocantilever model. The experiments show that the performances of the observer are convenient for micromanipulation/microassembly tasks.
\end{abstract}

\section{INTRODUCTION}

In micromanipulation/microassembly, piezoelectric materials are very widespread because of the rapidity and the high resolution that they can offer. There are many piezoelectric materials based applications in micromanipulation/microassembly but one of the most used to manipulate a micro-obect is microgripper (examples in [1][2]). A piezoelectric microgripper is composed of two piezoelectric cantilevers (piezocantilevers) (Fig. 1). When an electrical excitation is applied to each piezocantilever, it bends. The main objective is to pick the micro-object, transport or place it precisely to an other position thanks to the deflections of the cantilevers. If the manipulated object

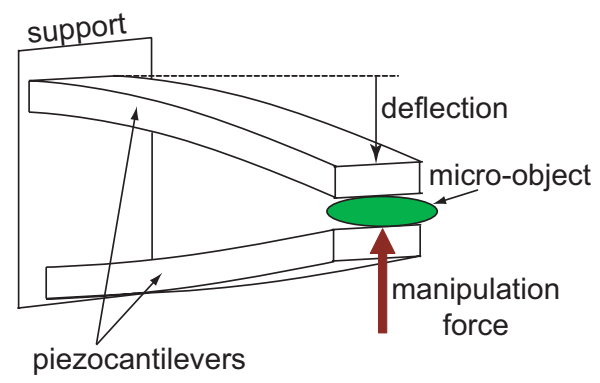

Fig. 1. A piezoelectric microgripper made up of two piezocantilevers.

is fragile (optical objects, biological objects, etc.), it is important to measure and control the manipulation

FEMTO-st Institute,

UMR CNRS-6174 / UFC / ENSMM / UTBM

Automatic Control and Micro-Mechatronic Systems department (AS2M department)

25000 Besançon - France

\{mrakoton, plutz\}@femto-st.fr force. Conversely, if the manipulated object is rigid, the piezocantilevers might break if the force is not controlled. Therefore, a concern application is the accurate positionning of the object with the first piezocantilever while the force measurement and control with the second one [3].

Unfortunately, while the modelling and control of the deflection or strain of piezoelectric materials are enough present in the litterature ([4] gives a survey), those of the force are not. This is mainly due to the lack of force sensors convenient for microsystems and micromanipulation/microassembly either on performances or on sizes. Hence, instead of measuring the force, an alternative technique is to observe it using the available signals. In his $\mathrm{PhD}$ thesis [5], Haddab uses the linear relation between the induced charges and the force to reconstruct the latter. However, the technique requires a good conditionning setup to well recover a good signal. In addition, the technique limits the piezocantilevers to only works in sensing, not in both sensing/actuating. To overcome these limitations, the same litterature [5] proposes to consider the force as a state variable with a null derivative and to observe it thanks to a linear discrete observer. The input signals of the observer are the applied voltage and the measured deflection. Despite the convergency of such closed-loop kind technique, it requires the dynamic part of the force. Although, it has been demonstrated that the dynamic part of the manipulation force always depends on the characteristic of the manipulated object either for classical manipulators [6] or for piezocantilevers manipulators [7]. It is obvious that identifying them for each change of objects is not cogent. To avoid the use of the object characteristics in the modelling and observation, an open-loop kind observer has been proposed in our previous work [8]. As the technique requires a high accurate model, it is not anough robust relative to model errors and does not ensure convergency.

The core of this paper is the observation of the manipulation force between a piezocantilever and manipulated objects. To avoid the use of the object characteristics and to ensure convergency, we consider the force as an unknown input and we use an Unknown Input Observation (UIO) technique. We especially use the InverseDynamics-Based UIO proposed by Liu and Peng [9] because their analyzed class of models are well suited for piezocantilevers. Basically, the goal is the estimation of the state and of the unknown input (force) of the 
piezocantilever.

First, the UIO observer technique used in this paper is presented. Then, we give the model of the piezocantilever. After that, the computation of the observer is presented. Finally, some experimental results of the force estimation end the paper. In the sequel, the term observation and estimation are alternated to mean the same signification.

\section{The Inverse-Dynamics-Based UIO technique}

We present in this section the Inverse-Dynamics-Based UIO technique to estimate both the state and the unknown input of a system [9].

\section{A. Problem statement}

Consider the following class of models:

$$
\begin{aligned}
& \dot{x}=A x+\Gamma(u, y)+B d \\
& y=C x
\end{aligned}
$$

where $x \in R^{n}$ denotes the state vector, $y \in R^{n_{y}}$ denotes the output vector, $u \in R^{n_{u}}$ denotes the input vector, $\Gamma(u, y) \in R^{n}$ is a known nonlinear function of $u$ and $y$, $d \in R^{n_{d}}$ is the unknown input, $A \in R^{n \times n}$ is the state matrix, $C \in R^{n_{y} \times n}$ is the output matrix and $B \in R^{n \times n_{d}}$ is the disturbance input matrix. For SISO problem, we have $n_{y}=n_{d}=1$. $n_{u}$ may be different from one. Indeed the studied problem is a disturbance estimation rather than a control problem. In the sequel, the unknown input is also called disturbance.

The following assumptions are assumed:

- the matrices $A, B$ and $C$ are known,

- $B$ has full column rank,

- $(A, C)$ is observable.

The objective is to simultaneously estimate $x$ and $d$ from the known signals $y$ and $u$.

\section{B. The equations of the observer}

The equation of the state observer is:

$$
\begin{aligned}
& \dot{\hat{x}}=A \hat{x}+\Gamma(u, y)+B \hat{d}+K(y-\hat{y}) \\
& \hat{y}=C \hat{x}
\end{aligned}
$$

and the equation of the disturbance observer is:

$$
\hat{d}=F_{1} y+F_{2} \dot{y}+G_{1} \hat{x}+G_{2} \dot{\hat{x}}+G_{3} \Gamma(u, y)
$$

where:

- the symbol ^ denotes estimation,

- $K$ is the gain of the state obsever,

- $F_{1} \in R^{n_{d} \times n_{y}}, F_{2} \in R^{n_{d} \times n_{y}}, G_{1} \in R^{n_{d} \times n}, G_{2} \in$ $R^{n_{d} \times n}$ and $G_{3} \in R^{n_{d} \times n_{y}}$ are gains of the disturbance observer.

The error dynamics is obtained using (equ 1), (equ 2) and (equ 3):

$$
\dot{e}=(A-K C) e+B e_{d}
$$

with $e=x-\hat{x}$ and $e_{d}=d-\hat{d}$.

The issue is to find the convenient gains $K, F_{1}, F_{2}$, $G_{1}, G_{2}$ and $G_{3}$ to make $e$ and $e_{d}$ converge exponentially to zero. Depending on whether there exists $F_{2}$ or not such as $F_{2} C B-I_{n_{d} \times n_{d}}=0$, two observer schemes were proposed.

\section{First observer scheme}

There exists $F_{2}$ so that $F_{2} C B-I_{n_{d} \times n_{d}}=0$. For SISO problem, this is satisfied if and only if $C B \neq 0$. Thus:

(i) $F_{2}$ is chosen to satisfy

$$
F_{2} C B-I_{n_{d} \times n_{d}}=0
$$

(ii) $F_{1}$ and $K$ are selected such as

$$
A-B\left(F_{1} C+F_{2} C A\right)-K C
$$

is Hurwitz

(iii) and

$$
\begin{aligned}
& G_{1}=-\left(F_{1} C+F_{2} C A\right) \\
& G_{2}=0 \\
& G_{3}=-F_{2} C
\end{aligned}
$$

Indeed, using these gains, the disturbance error becomes dependent of the state error:

$$
e_{d}=-\left(F_{1} C+F_{2} C A\right) e
$$

and the error dynamic described by (equ 4) becomes:

$$
\dot{e}=\left(A-B\left(F_{1} C+F_{2} C A\right)-K C\right) e
$$

Because of the (ii) condition, the error dynamic is exponentially stable and $e$ tends towards zero.

\section{Second observer scheme}

Many physical systems fail to satisfy the condition required for the precedent observer scheme. Hence, if for any $F_{2}$ one cannot satisfy $F_{2} C B-I_{n_{d} \times n_{d}}=0$, a second observer scheme was proposed.

Let $B^{+}$be the Penrose-Moore inverse of $B$. If one chooses:

$$
\begin{aligned}
& G_{1}=-\left(F_{1} C+B^{+} A\right) \\
& G_{2}=-\left(F_{2} C-B^{+}\right) \\
& G_{3}=-B^{+}
\end{aligned}
$$

the error dynamic described by (equ 4) becomes:

$$
M_{e} \dot{e}=A_{e} e
$$

where $M_{e}=I+B\left(F_{2} C-B^{+}\right)$and $A_{e}=A-$ $B\left(F_{1} C+B^{+} A\right)-K C$.

If $M_{e}$ is nonsingular, the gains $F_{1}, F_{2}$ and $K$ should be selected such as $M_{e}^{-1} A_{e}$ is Hurwitz. However if $M_{e}$ is singular, the singular value decomposition (SVD) is used. Let:

$$
\begin{aligned}
& M_{e}=U \Sigma V^{t} \\
& \Sigma=\left[\begin{array}{ll}
\sigma & 0 \\
0 & 0
\end{array}\right]
\end{aligned}
$$

where $U \in R^{n \times n}$ and $V \in R^{n \times n}$ are unitary matrices, and $\sigma \in R^{n_{m} \times n_{m}}\left(n_{m} \leq n\right)$ is a positive-definite diagonal matrix. Using the (equ 11) and (equ 12), we obtain:

$$
\left[\begin{array}{ll}
\sigma & 0 \\
0 & 0
\end{array}\right]\left[\begin{array}{c}
\dot{z}_{1} \\
\dot{z}_{2}
\end{array}\right]=\left[\begin{array}{ll}
A_{11} & A_{12} \\
A_{21} & A_{22}
\end{array}\right]\left[\begin{array}{l}
z_{1} \\
z_{2}
\end{array}\right]
$$


with:

$$
\begin{aligned}
& z=\left[\begin{array}{l}
z_{1} \\
z_{2}
\end{array}\right] \equiv V^{t} e \\
& {\left[\begin{array}{ll}
A_{11} & A_{12} \\
A_{21} & A_{22}
\end{array}\right] \equiv U^{t} A_{e} V}
\end{aligned}
$$

Then, the error dynamic finally becomes:

$$
\begin{aligned}
& \dot{z}_{1}=\sigma^{-1} A_{11} z_{1}+\sigma^{-1} A_{12} z_{2} \\
& 0=\sigma^{-1} A_{21} z_{1}+\sigma^{-1} A_{22} z_{2}
\end{aligned}
$$

The core of the second observer scheme is to ensure the exponential stability of (equ 15). For that, $K, F_{1}$ and $F_{2}$ should be selected such as $A_{22}$ and $A_{11}-A_{12} A_{22}^{-1} A_{21}$ are Hurwitz.

\section{Model OF THE PIEZOCANTILEVER}

\section{A. Presentation of the setup}

The experimented piezocantilever is made up of a piezolayer and a Copper layer. Its sizes are: $15 \mathrm{~mm} \times$ $2 m m \times 300 \mu m$ (active length, width and height). The identification and the estimation experiments are performed using the Matlab-Simulink (c) software and a computer-DSpace hardware with $0.2 \mathrm{~ms}$ of sampling time. A Keyence optical sensor is used to measure the deflection. It has $10 \mathrm{~nm}$ of resolution and better than $400 \mu \mathrm{m}$ of accuracy. As there is no convenient sensor to check the force, the experiments are performed with known weights applied at the tip of the piezocantilever (Fig. 2).

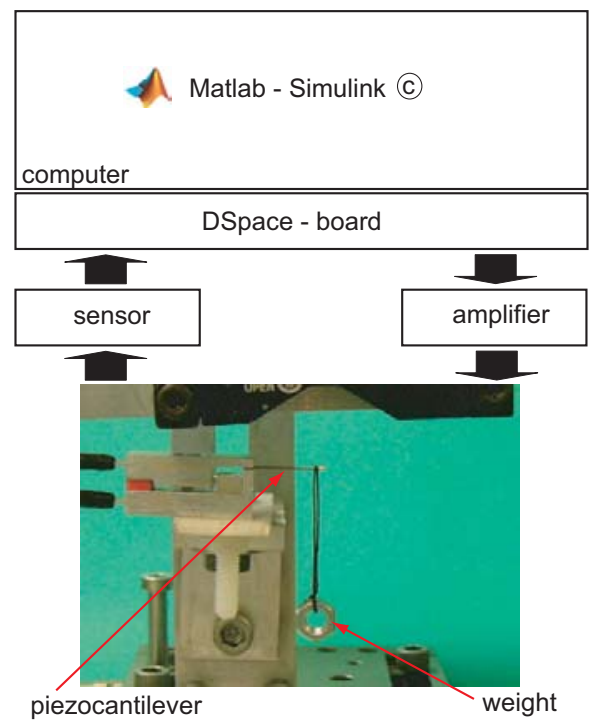

Fig. 2. The setup.

\section{B. The state-space model}

Let $\delta$ be the deflection at the tip of the cantilever, $U$ the applied voltage and $F=d$ the unknown applied force. When the applied electrical field is low, the piezocantilever behavior is linear (example in [3]). We have:

$$
\left\{\begin{array}{l}
{\left[\begin{array}{l}
\dot{x}_{1} \\
\dot{x}_{2}
\end{array}\right]=\left[\begin{array}{ll}
a_{1} & a_{2} \\
a_{3} & a_{4}
\end{array}\right]\left[\begin{array}{l}
x_{1} \\
x_{2}
\end{array}\right]+\left[\begin{array}{l}
\alpha \\
0
\end{array}\right] U+\left[\begin{array}{l}
\beta \\
0
\end{array}\right] F} \\
y=\delta=\left[\begin{array}{ll}
0 & c
\end{array}\right]\left[\begin{array}{l}
x_{1} \\
x_{2}
\end{array}\right]
\end{array}\right.
$$

where the identified parameters are given by the Table I. The identification process was as follow:

- a step voltage input $U$ is first applied to the piezocantilever,

- afterwards, a mass with known weight $F$ is hung on at its tip,

- using the input data $U$ and the read-out $\delta$, an ARMAX transfer function model is first identified and then transformed into the state-space model described by (equ 16). The identified model is without the elastic gain $\beta$. The latter is identified using the input data $F$ and the corresponding output $\delta$.

In our model, we have $B=\left[\begin{array}{ll}\beta & 0\end{array}\right]^{t}$.

TABLE I

Identified parameters.

\begin{tabular}{|l|l||l|l|}
\hline \hline parameters & values & parameters & values \\
\hline$a_{1}$ & -1333.3 & $\alpha$ & 3.856 \\
\hline$a_{2}$ & -162.8 & $\beta$ & 56.8 \\
\hline$a_{3}$ & 2048 & $c$ & 10.2 \\
\hline$a_{4}$ & 0 & & \\
\hline \hline
\end{tabular}

Fig. 3 presents the simulation of the model and the experimental results when a step voltage with $10 \mathrm{~V}$ of amplitude is applied.

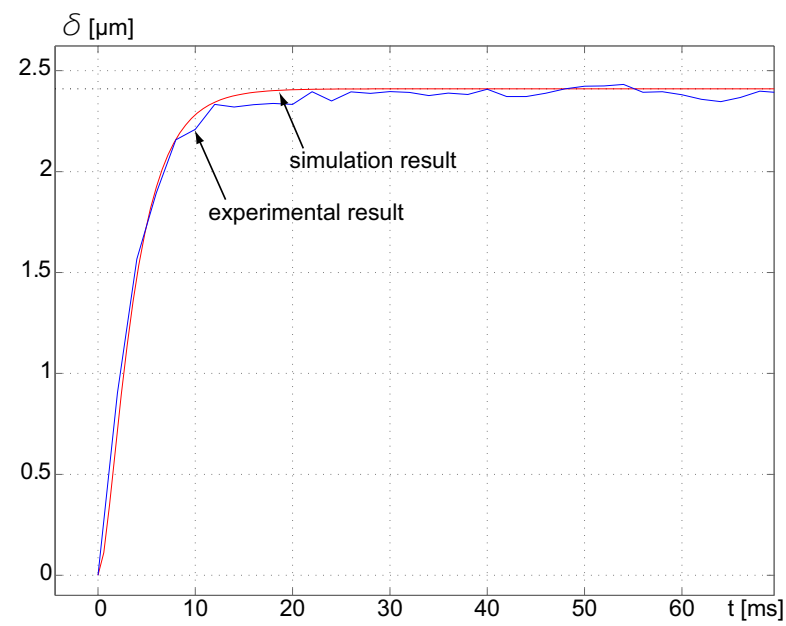

Fig. 3. Step response of the piezocantilever. 


\section{Computation of the OBSERVER}

\section{A. Assumptions verification}

The necessary conditions to use the Inverse-DynamicsBased UIO technique are satisfied. Indeed:

(i) comparing the model in (equ 1) and the piezocantilever model in (equ 16), the matrices $A, B$ and $C$ are known.

(ii) $\operatorname{rank}(B)=1$ is full.

(iii) $\operatorname{rank}\left(\left[\begin{array}{c}C \\ C A\end{array}\right]\right)=2$ is full.

\section{B. Computation of the gains}

As $C B=0$, the second observer scheme is used in this paper. Consider the Penrose-Moore inverse of $B$ :

$$
B^{+}=\left[\begin{array}{cc}
\frac{1}{\beta} & \rho
\end{array}\right]
$$

where any $\rho \in R$ is convenient. Hence, the matrices $M_{e}$ and $A_{e}$ can be deduced:

$$
\begin{aligned}
& M_{e}=\left[\begin{array}{cc}
0 & \beta\left(F_{2} c-\rho\right) \\
0 & 0
\end{array}\right] \\
& A_{e}=\left[\begin{array}{cc}
-\rho \beta a_{3} & -k_{1} c-\beta F_{1} c-\rho \beta a_{4} \\
a_{3} & a_{4}-k_{2} c
\end{array}\right]
\end{aligned}
$$

Because $M_{e}$ is singular, we consider its SVD. We have:

$$
\begin{aligned}
& U=\frac{1}{\sqrt{\beta^{2}\left(F_{2} c-\rho\right)^{2}+1}}\left[\begin{array}{cc}
\beta\left(F_{2} c-\rho\right) & 1 \\
1 & -\beta\left(F_{2} c-\rho\right)
\end{array}\right] \\
& \sum=\left[\begin{array}{cc}
\sqrt{\beta^{2}\left(F_{2} c-\rho\right)^{2}+1} & 0 \\
0 & 0
\end{array}\right] \\
& V=\left[\begin{array}{ll}
0 & 1 \\
1 & 0
\end{array}\right]
\end{aligned}
$$

So:

$$
\begin{aligned}
& U^{t} A_{e} V=\left[\begin{array}{ll}
A_{11} & A_{12} \\
A_{21} & A_{22}
\end{array}\right]=\frac{1}{\sqrt{\beta^{2}\left(F_{2} c-\rho\right)^{2}+1}}\left[\begin{array}{ll}
\theta_{11} & \theta_{12} \\
\theta_{21} & \theta_{22}
\end{array}\right] \\
& \theta_{11}=-\beta\left(F_{2} c-\rho\right)\left(k_{1}+\beta F_{1} c+\rho \beta a_{4}\right)+a_{4}-k_{2} c \\
& \theta_{12}=-\rho \beta^{2} a_{3}\left(F_{2} c-\rho\right)+a_{3} \\
& \theta_{21}=-\left(k_{1}+\beta F_{1} c+\rho \beta a_{4}\right)-\beta\left(F_{2} c-\rho\right)\left(a_{4}-k_{2} c\right) \\
& \theta_{22}=-\beta F_{2} c a_{3}
\end{aligned}
$$

The matrices $A_{22}$ and $A_{11}-A_{12} A_{22}^{-1} A_{21}$ are respectively Hurwitz if:

$$
\beta F_{2} c a_{3}>0
$$

and

$$
\begin{aligned}
& \rho^{3} \beta^{3} k_{2} c+\rho^{2} \beta^{2}\left(\beta F_{1} c-2 \beta F_{2} k_{2} c^{2}+k_{1}\right) \\
& +\rho \beta c\left(\beta^{2} F_{2}^{2} k_{2} c^{2}-2 \beta^{2} F_{1} F_{2} c-2 \beta F_{2} k_{1}+k_{2}\right) \\
& +\left(\beta^{3} F_{1} F_{2}^{2} c^{3}+\beta^{2} F_{2}^{2} k_{1} c^{2}+\beta F_{1} c+k_{1}\right)>0
\end{aligned}
$$

In addition to $K, F_{1}$ and $F_{2}, \rho$ is an extra design degree of freedom. There is no systematic method to directly choose optimal values of theses parameters.
Moreover, there may be gains that satisfy (inequ 22) and (inequ 21) but which derive an unstability of the observer. So, a simulation verification (with Simulink (C) is used to enforce their choice Fig. 4. In the figure, the PIEZOCANTILEVER bloc contains the state-space model described by (equ 16), the STATE-observer bloc contains the (equ 2) and the FORCE-observer bloc contains the (equ 3). Because of an algebraic loop problem, we introduce a filter with a large bandwidth in the observer connection.

The simulation is processed as follow. At $t=0$, a step voltage $U=10 \mathrm{~V}$ is applied to the piezocantilever. Then, at respectively $t=20 \mathrm{~ms}$ and $t=40 \mathrm{~ms}$, a force of $F=2 m N$ and $F=6 m N$ are applied. The simulation results (Fig. 5) show that the estimate deflection and force immediatly coincide with the output deflection and applied force. Besides the conditions in (inequ 22) and (inequ 21), the gains are chosen such as the convergence (settling) time of the force observer $(\approx 9 m s)$ is less or equal to the settling time of the piezocantilever $(\approx$ $10 \mathrm{~ms}$ ). The vibrations in the estimate force (Fig. 5-b) can be reduced by adjusting the observer gain but the settling time would increase.

We find:

$$
\begin{aligned}
& \rho=0.045 \quad K=\left[\begin{array}{c}
47 \\
0
\end{array}\right] \quad \begin{array}{c}
F_{1}=0.335 \\
G_{1}
\end{array}=\left[\begin{array}{ll}
-68.69 & -0.542
\end{array}\right] \\
& F_{2}=8.18 \times 10^{-3} \quad\left[\begin{array}{ll}
0.0176 & -0.038
\end{array}\right] \\
& G_{2}=\left[\begin{array}{ll}
-0.0176 & -0.045
\end{array}\right]
\end{aligned}
$$
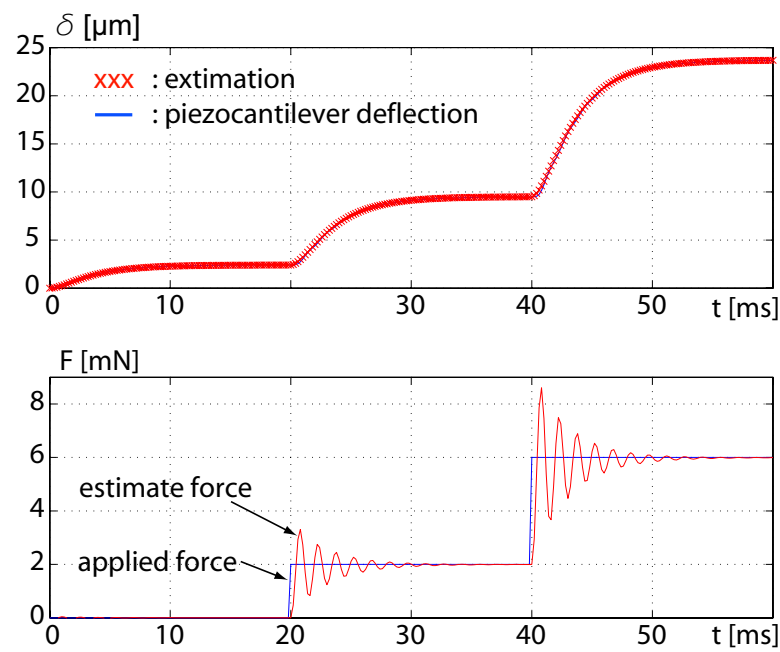

Fig. 5. Simulation results of the force estimation.

\section{EXPERIMENTS}

The observer has been implemented in the computerDSpace material. The aim of the experiments is to estimate a weight applied at the piezocantilever tip for different voltages. 


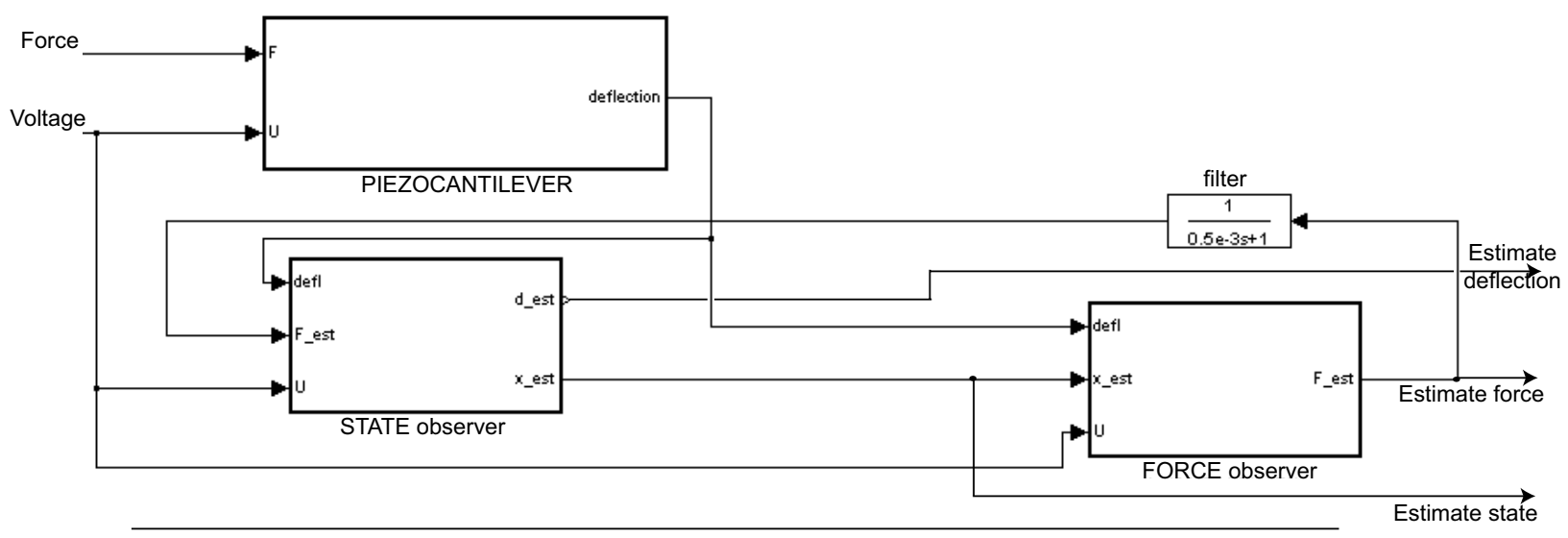

Fig. 4. Simulation of the observer.

A. Estimation of a null force
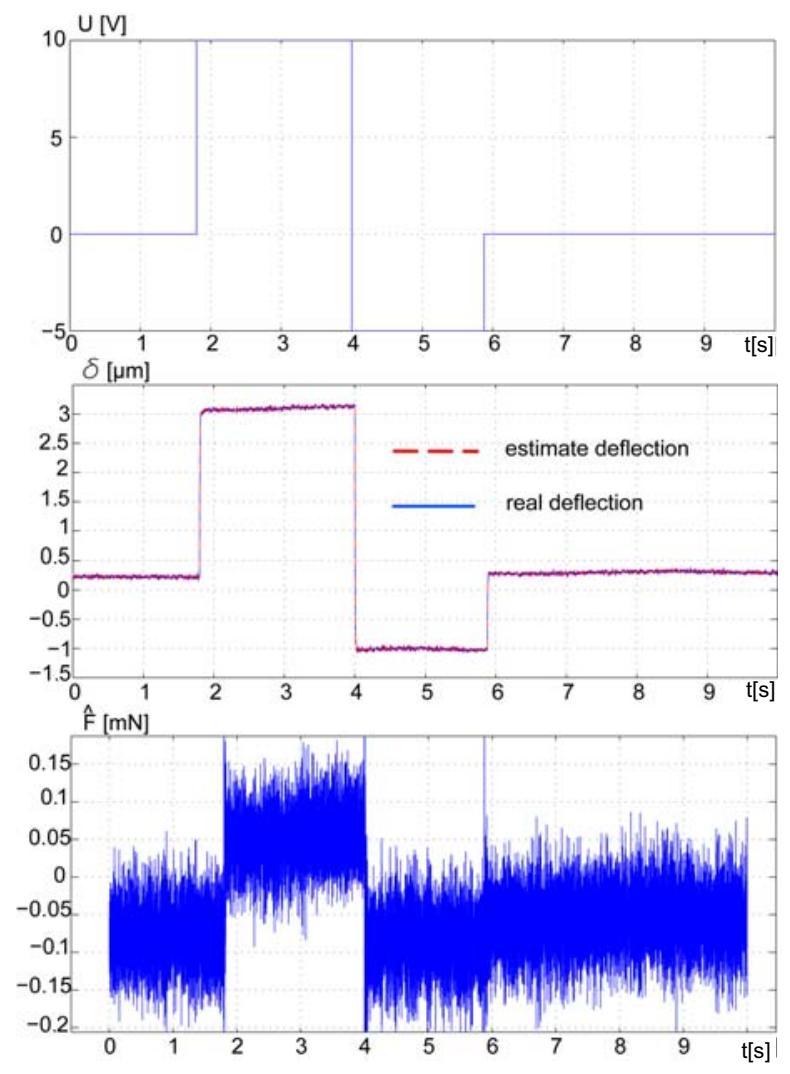

Fig. 6. Estimation of a null force. a: the applied voltage. b: the deflection (real and estimate). c: the estimate force.

First, no weight is put at the tip of the piezocantilever while two electrical steps are applied at different times. The results (Fig. 6) show the results. On the one hand, the estimate deflection and the real deflection well coincide. On the other hand, besides the accuracy of the sensor, the influence of the voltage into the estimate force is insignificant. Indeed, the corresponding accuracy is better than $0.015\left[\frac{m N}{V}\right]$ (computed using the figures
Fig. 6-a and Fig. 6-c).

\section{B. Estimation of a non-null force}

Next, a weight with $1.6 \mathrm{~m} N$ amplitude is put at the tip. In the same way, different steps voltage are applied. The results are shown in the Fig. 7 . Because of the difficulty to put brusquely the weight, the force signal is not a real step. Hence, there are low dynamic and vibration either in the resulting deflection or in the estimate force. These vibrations can be seen in the deflection and force when putting the mass at nearly $t=4 s$ (Fig. 7). Despite this conditionning matter, the relative accuracy of the estimate force is interesting: $6.25 \%\left(\approx \frac{0.15[\mathrm{mN}]}{1.6[\mathrm{mN}]}\right.$, computed from the Fig. 7-c and Fig. 7-d. Indeed, the provided accuracy is still sub-milliNewton even covering the forces used in micromanipulation/microassembly tasks that are up to a few tens of milliNewton. However, it is clear that the resolution will be limited by the deflection sensor and the stiffness of the piezocantilever.

C. Estimation of a non-null force with harmonic electrical excitation

The last experiment concerns the use of harmonic electrical input. The aim is to evaluate the invariancy of the estimate force relative to variable input voltage. To perform the experiment, a weight $(F=1.6 \mathrm{mN})$ is hung at the tip while a sine voltage is applied. The results are given in Fig. 8. The deflection magnitude is given by $20 \log \left(\frac{\delta_{a m p}}{U_{a m p}}\right)$ and the force magnitude is defined as $20 \log \left(\frac{\hat{F}_{a m p}}{F}\right)$, where the subscript amp means amplitude of the sine signal. It clearly appears that the force observer is independent of the frequency of the input voltage, at least up to the concerned bandwidth. The fact that the force magnitude is non exactly equal to $0 d B$ is due to the accuracy as spoken in the previous subsection.

\section{Conclusion}

This paper has presented the estimation of the force applied at the tip of a piezocantilever. The final objective 
is to observe and control the manipulation force between a microgripper and a micro-object during micromanipulation/microassembly tasks.

In our approach, the force has been considered as an Unknown Input. Thus, we have used an Unknown Input Observation (UIO) technique to estimate it. Indeed, this avoids the use of the characteristics of the manipulated objects which are difficult and time-consumming to obtain. The Inverse-Dynamics-Based UIO technique was especially used because it is well suited for the model of a piezocantilever. The experimental results shown that the performances provided by the proposed estimation is suitable for micromanipulation/microassembly tasks. Among others, the accuracy is sub-milliNewton when the
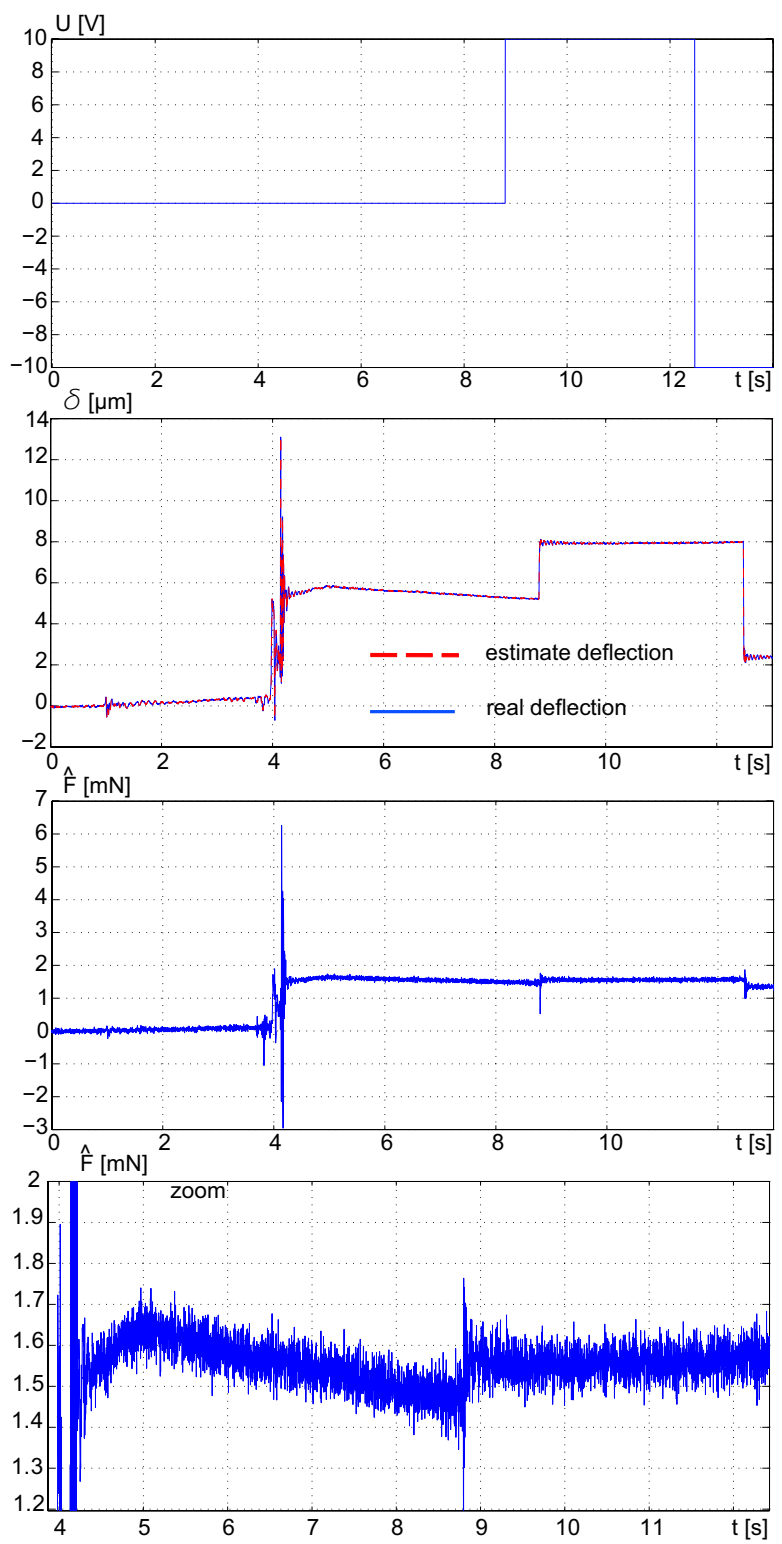

Fig. 7. Estimation of a non-null force $(1.6 \mathrm{mN})$. a: the applied voltage. b: the deflection (real and estimate). c: the estimate force. $\mathrm{d}$ : zoom of the estimate force.

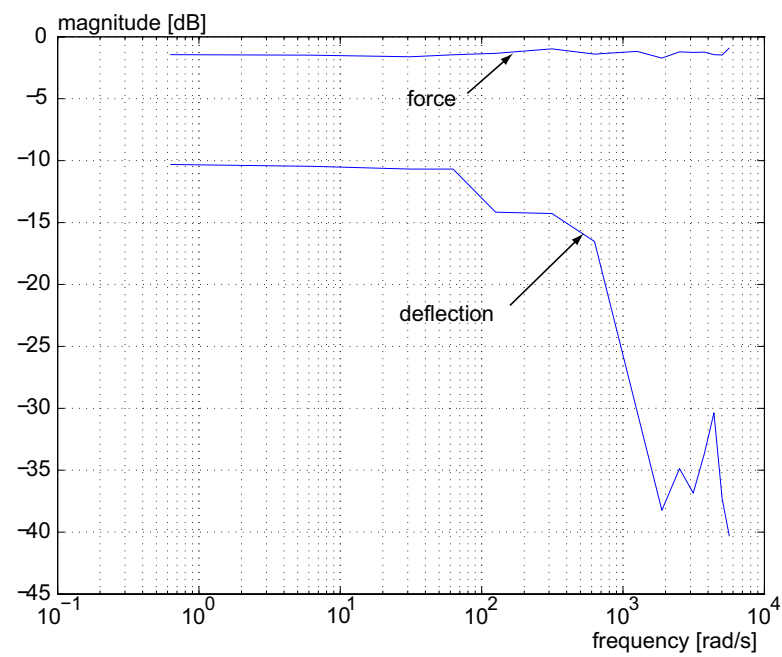

Fig. 8. Experiment with harmonic input voltage.

range of estimation is some tens of milliNewton.

The future works will be the integration of the observer into a complete microgripper and the control of force/position through the measured/estimated signals.

\section{ACKNOWLEDGMENT}

This work is partially supported by the French research project NANOROL (ANR $N^{\circ}$ PSIROB07_184846).

\section{REFERENCES}

[1] Y. Haddab, N. Chaillet and A. Bourjault, 'A microgripper using smart piezoelectric actuators', IEEE/RSJ IROS, Takamatsu Japan, October-November 2000.

[2] J. Agnus, J. M. Breguet, N. Chaillet, O. Cois, P. de Lit, A. Ferreira, P. Melchior, C. Pellet and J. Sabatier, 'A smart microrobot on chip: design, identification and modeling', IEEE/ASME AIM, Kobe Japan, pp.685-690, 2003.

[3] M. Rakotondrabe, C. Clévy and P. Lutz, 'Modelling and robust position/force control of a piezoelectric microgripper', IEEE CASE, Scottsdale AZ USA, pp.39-44, September 2007.

[4] S. Devasia, E. E. Eleftheriou, R. Moheimani, 'A survey of control issues in nanopositioning' IEEE Transactions on Control Systems Technology, Vol.15, No15, pp.802-823, 2007.

[5] Y. Haddab, 'Design and development of a micromanipulation system with position/force control and dedicated to micrometric objects', PhD thesis in Automatic Control and Computer Science, Université de Franche-Comté, 2000.

[6] S. D. Eppinger and W. P. Seering, 'On dynamic models of robot force control', IEEE ICRA, pp.29-34, April 1986.

[7] M. Rakotondrabe, Y. Haddab and P. Lutz 'Modelling and H-inf force control of a nonlinear piezoelectric cantilever', IEEE/RSJ IROS, San Diego CA USA, pp.3131-3136, October November 2007.

[8] M. Rakotondrabe, Y. Haddab and P. Lutz 'Nonlinear modelling and estimation of force in a piezoelectric cantilever', IEEE/ASME - AIM, Zurich Switzerland, September 2007.

[9] C-S. Liu and H. Peng, 'Inverse-dynamics based state and disturbance observers for linear time-invariant systems', ASME Journal of Dynamics Systems, Measurement and Control, vol.124, pp.375-381, September 2002. 